\title{
Erratum: Multiphonon excitations from dark matter scattering in crystals [Phys. Rev. D 101, 036006 (2020)]
}

\author{
Brian Campbell-Deem $\odot$, Peter Cox, Simon Knapen, Tongyan Lin, and Tom Melia
}

(Received 23 June 2020; published 28 July 2020)

DOI: 10.1103/PhysRevD.102.019904

In the original evaluation for the paper, a second term given by perturbation theory in the anharmonic rate calculation was not included. Thus, Eq. (A6) for the transition rate is modified to include this term (last line of the equation below),

$$
\begin{aligned}
w_{i \rightarrow f}= & 2 \pi \delta\left(\omega_{\nu_{1}, \mathbf{k}_{1}}+\omega_{\nu_{2}, \mathbf{k}_{2}}-\omega\right) \\
& \times \mid\left\langle p_{f} ; \nu_{1}, \mathbf{k}_{1} ; \nu_{2}, \mathbf{k}_{2}|\mathcal{V}(\mathbf{r})| p_{i} ; 0\right\rangle+\sum_{\nu, \mathbf{k}}\left(\frac{\left\langle p_{f} ; \nu, \mathbf{k}|\mathcal{V}(\mathbf{r})| p_{i} ; 0\right\rangle\left\langle\nu_{1}, \mathbf{k}_{1} ; \nu_{2}, \mathbf{k}_{2}|\delta H| \nu, \mathbf{k}\right\rangle}{\omega-\omega_{\nu, \mathbf{k}}+i \Gamma_{\nu, \mathbf{k}} / 2}\right. \\
& \left.+\frac{\left\langle\nu, \mathbf{k} ; \nu_{1}, \mathbf{k}_{1} ; \nu_{2}, \mathbf{k}_{2}|\delta H| 0\right\rangle\left\langle p_{f} ; 0|\mathcal{V}(\mathbf{r})| p_{i} ; \nu, \mathbf{k}\right\rangle}{-\omega-\omega_{\nu, \mathbf{k}}+i \Gamma_{\nu, \mathbf{k}} / 2}\right)\left.\right|^{2}
\end{aligned}
$$

With this correction, there is a cancellation between the last two terms, which causes the anharmonic structure factor to scale as $\sim q^{4}$ rather than $\sim q^{2}$. There are corresponding changes to Eqs. (13), (17), (22)-(26), and (46)-(47) for the anharmonic matrix element and structure factors.

The effect on the total multiphonon scattering rate is a decrease by $2-3$ orders of magnitude. The updated numerical structure factors and rates are reflected in Figs. 2 and 3, and the sensitivity in the dark matter cross section is also updated in Fig. 4. The conclusions of this paper, namely that multiphonons are subdominant to production of single optical or acoustic phonons for sub-MeV dark matter, are not changed. We give the detailed list of corrections below.

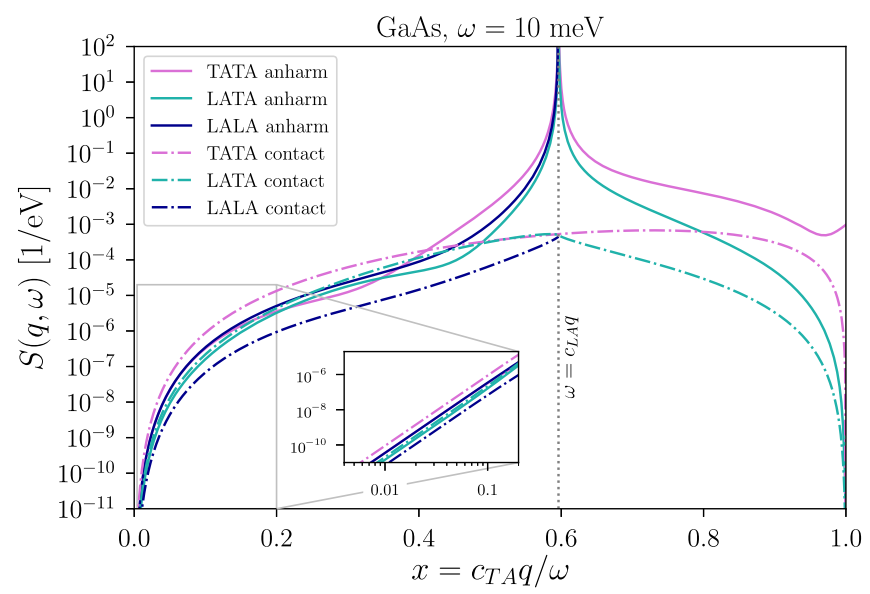

FIG. 2. The plot of the structure factors for each channel contains the updated anharmonic structure factors.

Published by the American Physical Society under the terms of the Creative Commons Attribution 4.0 International license. Further distribution of this work must maintain attribution to the author(s) and the published articles title, journal citation, and DOI. 

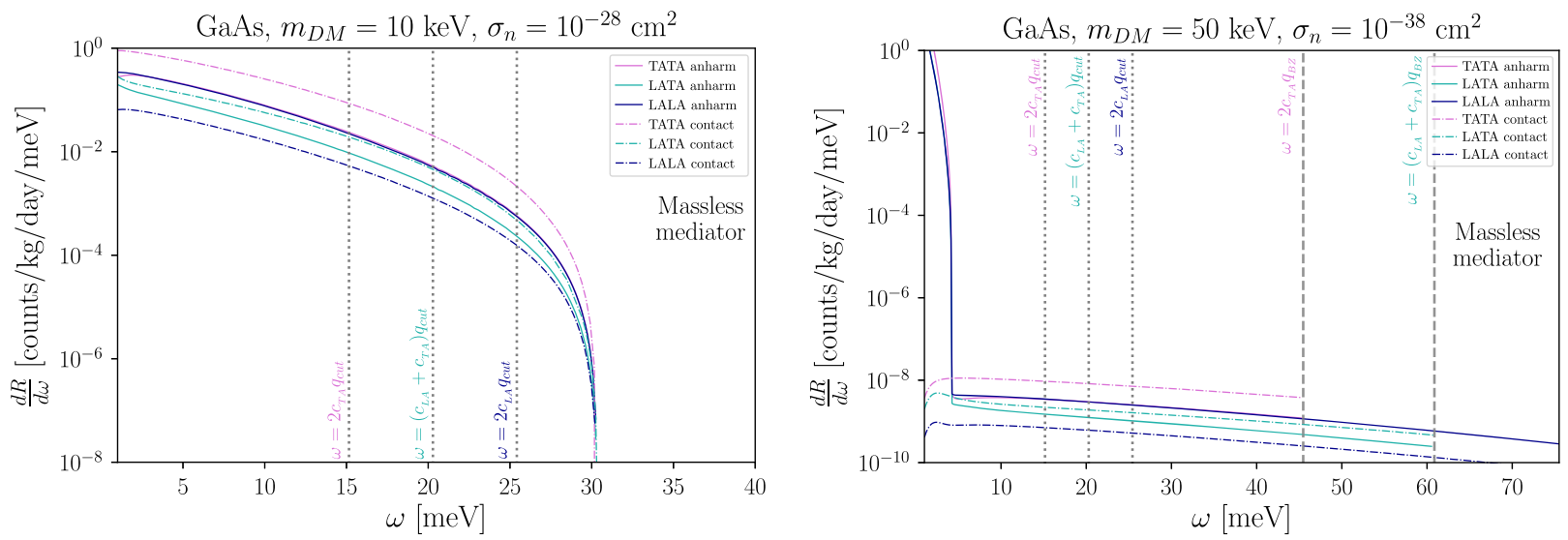

FIG. 3. The differential rate plots are updated with the new anharmonic structure factors.
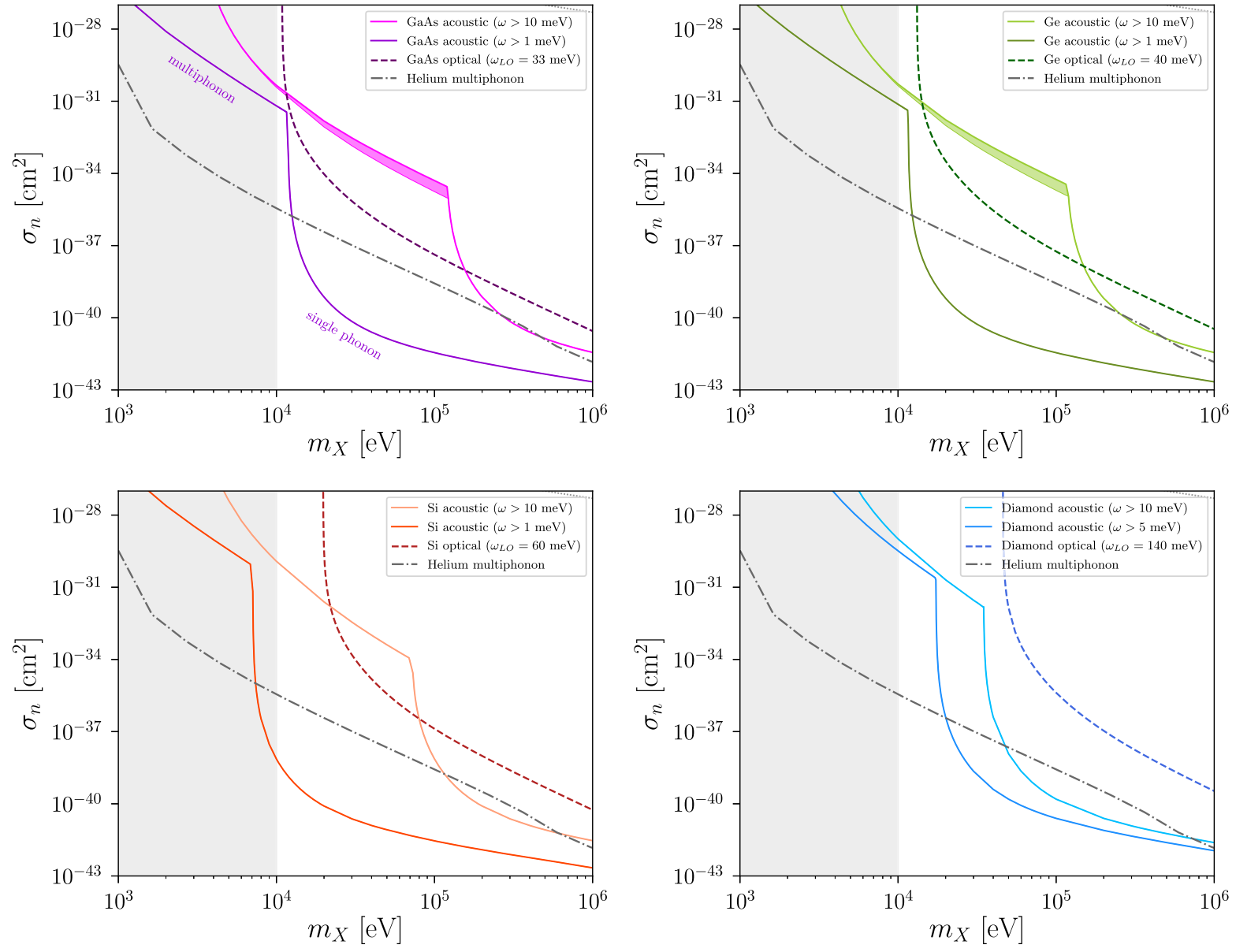

FIG. 4. The cross section sensitivity plots feature updated multiphonon lines. 
Table I: The low-q scaling of the anharmonic channel is now $\# q^{4}$,

Channel

Single acoustic phonon

Single optical phonon

Multiphonon (contact)

Multiphonon (anharmonic)

Multiphonon (helium)
Low- $q$ scaling

$q$
$q^{4}$
$q^{4}$
$\# q^{4}$
$\# q^{4}$

Equation 13: The anharmonic matrix element now includes the second term

$$
\begin{aligned}
\mathcal{M}_{f, \mathbf{q}, d}^{(a n h)}= & i \sum_{\mathbf{G , k}, \nu} \sqrt{\frac{N}{2 m_{d} \omega_{\nu, \mathbf{k}}}}\left(\frac{\mathbf{q} \cdot \mathbf{e}_{\nu, d, \mathbf{k}}^{*}\left\langle\nu_{1}, \mathbf{k}_{1} ; \nu_{2}, \mathbf{k}_{2}|\delta H| \nu, \mathbf{k}\right\rangle}{\omega_{\nu_{1}, \mathbf{k}_{1}}+\omega_{\nu_{2}, \mathbf{k}_{2}}-\omega_{\nu, \mathbf{k}}+i \Gamma_{\nu, \mathbf{k}} / 2} e^{i(\mathbf{q}-\mathbf{k}) \cdot \mathbf{r}_{d}^{0}} \delta_{\mathbf{G}, \mathbf{q}-\mathbf{k}}\right. \\
& \left.+\frac{\mathbf{q} \cdot \mathbf{e}_{\nu, d, \mathbf{k}}\left\langle\nu, \mathbf{k} ; \nu_{1}, \mathbf{k}_{1} ; \nu_{2}, \mathbf{k}_{2}|\delta H| 0\right\rangle}{-\left(\omega_{\nu_{1}, \mathbf{k}_{1}}+\omega_{\nu_{2}, \mathbf{k}_{2}}\right)-\omega_{\nu, \mathbf{k}}+i \Gamma_{\nu, \mathbf{k}} / 2} e^{i(\mathbf{q}+\mathbf{k}) \cdot \mathbf{r}_{d}^{0}} \delta_{\mathbf{G}, \mathbf{q}+\mathbf{k}}\right),
\end{aligned}
$$

Equation 17: The unevaluated anharmonic structure factor includes the second term,

$$
\begin{aligned}
S^{(a n h)}(\mathbf{q}, \omega)= & \frac{1}{16} \frac{\sum_{d} A_{d}}{m_{p} \rho^{3}} \sum_{\nu_{1}, \nu_{2}} \int \frac{d^{3} \mathbf{k}_{1}}{(2 \pi)^{3}} \mid \frac{q \tilde{\mathcal{M}}\left(\mathbf{q}, \mathbf{k}_{i}, \nu_{i}\right)}{\omega_{L A, \mathbf{q}} \sqrt{\omega_{\nu_{1}, \mathbf{k}_{1}} \omega_{\nu_{2}, \mathbf{q}-\mathbf{k}_{1}}}}\left(\frac{1}{\omega-\omega_{L A, \mathbf{q}}+i \Gamma_{L A, \mathbf{q}} / 2}\right. \\
& \left.+\frac{1}{-\omega-\omega_{L A, \mathbf{q}}+i \Gamma_{L A, \mathbf{q}} / 2}\right)\left.\right|^{2} \delta\left(\omega-\omega_{\nu_{1}, \mathbf{k}_{1}}-\omega_{\nu_{2}, \mathbf{q}-\mathbf{k}_{1}}\right),
\end{aligned}
$$

Equations 22-26 The general and evaluated anharmonic structure factors have an updated propagator resulting from the combination of both terms in the perturbation theory,

$$
\frac{1}{\left[\left(\omega-c_{\mathrm{LA}} q\right)^{2}+\Gamma_{\mathrm{LA}, q}^{2} / 4\right]} \mapsto \frac{4 c_{L A}^{2} q^{2}}{\left[\left(\omega^{2}-\left(c_{L A} q\right)^{2}\right)^{2}+\left(c_{L A} q\right)^{2} \Gamma_{L A, q}^{2}\right]}
$$

Equations 46 and 47: The optical-acoustic anharmonic structure factors contain analogous changes as the acousticacoustic anharmonic structure factors,

$$
\begin{gathered}
S_{L O L A}^{(a n h)}(q, \omega)=\frac{\gamma_{G}^{2}}{2 \pi^{2}} \frac{\omega_{L O}\left(A_{1}+A_{2}\right)}{\bar{c}^{2} c_{L A} \rho m_{p}} \frac{q^{4}\left(\omega-\omega_{L O}\right)^{3}}{\left(\omega^{2}-\left(c_{L A} q\right)^{2}\right)^{2}} \theta\left(\omega-\omega_{L O}\right), \\
S_{L O T A}^{(a n h)}(q, \omega)=\frac{\gamma_{G}^{2}}{\pi^{2}} \frac{\omega_{L O}\left(A_{1}+A_{2}\right)}{\bar{c}^{2} c_{T A} \rho m_{p}} \frac{c_{L A}^{2}}{c_{T A}^{2}} \frac{q^{4}\left(\omega-\omega_{L O}\right)^{3}}{\left(\omega^{2}-\left(c_{L A} q\right)^{2}\right)^{2}} \theta\left(\omega-\omega_{L O}\right) .
\end{gathered}
$$

To reflect the changes in the results, we also update the following text:

Page 2, Sec. I B: "The right-hand diagram in Fig. 1 only relies on the DM coupling to a single phonon and is therefore lower order in $q / \sqrt{m_{n} \omega}$. This process instead occurs via an off shell phonon and the phonon self-interactions, which arise from the anharmonicity of the crystal potential."

Updated text: "The right-hand diagram in Fig. 1 instead occurs via an off shell phonon and phonon self-interactions, which arise in part from the anharmonicity of the crystal potential. While this diagram only relies on the DM coupling to a single phonon and therefore appears to be lower order in $q / \sqrt{m_{N} \omega}$, there is an additional suppression in $q$ from the insertion of the phonon self-interaction."

"For both the single optical mode and the two-phonon contact interaction, $S(q, \omega)$ scales as $\sim q^{4}$, while for the anharmonic contribution, it scales more favorably as $\sim q^{2}$. However, the latter requires an insertion of the phonon selfinteraction, which also provides a suppression. [...] and the reach ends up being competitive with or better than the cubic crystals we considered." 
Updated text: "For the single optical mode and the two-phonon processes, $S(q, \omega)$ scales as $\sim q^{4}$. [...] and the reach therefore exceeds that of the cubic crystals we considered, under idealized experimental conditions."

Page 9, Sec. III C: "The inset zooms in on the low momentum region and shows the $\sim q^{2}$ and $\sim q^{4}$ scaling of the anharmonic and contact contributions, respectively."

Updated text: "The inset zooms in on the low momentum region and shows the $\sim q^{4}$ scaling of both multiphonon contributions."

Page 11, Sec. IV: "The anharmonic terms always dominate over the contact terms." (removed)

Page 13, Sec. V A: "Integrating the structure factor to obtain the total rate we find that, for all the materials we consider, the LO-LA scattering rate is 1-2 orders of magnitude smaller than the single optical rate, depending on the $q_{\text {cut }}$ used for the acoustic phonons. The LO-LA process becomes further suppressed relative to the single optical with increasing DM mass."

Updated text: "Integrating the structure factor to obtain the total rate we find that, for all the materials we consider, the LO-LA scattering rate is 4 to 5 orders of magnitude smaller than the single optical rate, where we again impose the $q_{\text {cut }}$ values in Table III on the acoustic phonons (relaxing this cut increases the LO-LA rate, but it always remains negligible)."

Page 13-14, Sec. V B: "Given that our results qualitatively differ from similar calculations in superfluid helium, it is worthwhile to compare the symmetries of both systems in a bit more detail. Crystals spontaneously [...] scaling in the low $q$ limit. Schematically, the matrix element for three acoustic phonons in a crystal is therefore always of the form,

$$
\left|\mathcal{M}_{\text {crys }}\right|^{2} \sim \mid(\mathbf{q} \cdot \mathbf{e})\left(\mathbf{k}_{1} \cdot \mathbf{e}_{1}\right)\left(\mathbf{k}_{2} \cdot \mathbf{e}_{2}\right)+\text { permutations }\left.\right|^{2} \sim q^{2} .
$$

Superfluid helium on the other hand [...] where the second $\sim$ follows from momentum conservation $\left(\mathbf{q}=\mathbf{k}_{1}+\mathbf{k}_{2}\right)$. The key point here is that $[\ldots]$ phonon self-couplings in helium."

Updated text: "Here, we briefly compare our results with similar calculations of multiphonon production in superfluid helium. While the symmetries of the systems are different, in both cases the structure factor scales as $q^{4}$ in the limit $q \ll \omega$. Crystals spontaneously [...] scaling in the low $q$ limit. Combined with the $q$-dependent DM-phonon coupling, the resulting matrix element goes as $|\mathcal{M}|^{2} \sim q^{4}$. Superfluid helium on the other hand [...] where the second $\sim$ follows from momentum conservation $\left(\mathbf{q}=\mathbf{k}_{1}+\mathbf{k}_{2}\right)$. Despite the differences in symmetries, the scaling of the dynamic structure factor for phonons in superfluid helium is the same as for longitudinal acoustic phonons in crystals. However, the multiphonon rate in helium exceeds that in the crystals we considered (see Fig. 4), due to the stronger phonon self-couplings in helium."

Page 14, Sec. VI: "despite the less favorable scaling of the structure factor in a low-momentum expansion." (removed) 